\title{
Mid-IR observations of circumstellar disks ${ }^{\star}$
}

\section{Pre-main sequence objects}

\author{
O. Schütz ${ }^{1}$, G. Meeus ${ }^{2}$, and M. F. Sterzik ${ }^{3}$ \\ 1 Max-Planck-Institut für Astronomie, Königstuhl 17, 69117 Heidelberg, Germany \\ e-mail: schuetz@mpia.de \\ 2 Astrophysikalisches Institut Potsdam, An der Sternwarte 16, 14482 Potsdam, Germany \\ 3 European Southern Observatory, Alonso de Cordova 3107, Santiago 19, Chile
}

Received 17 June 2004 / Accepted 21 September 2004

\begin{abstract}
We present new $N$-band photometry and spectroscopy for a sample of eight pre-main sequence stars including T Tauri, Herbig Ae/Be stars and FU Ori objects using the ESO TIMMI2 camera at the La Silla observatory (Chile). For some objects this is their first $N$-band spectroscopic observation ever. The FU Ori stars V 346 Nor, V 883 Ori and Z CMa show a broad absorption band which we attribute to silicates, while for BBW 76 we find silicate emission. A comparison with ISO-SWS spectra of V 346 Nor and Z CMa taken in 1996/1997 reveals no differences in spectral shape. All T Tauri and Herbig Ae/Be stars possess $N$-band emission features. We model the emission spectra with a mixture of silicates consisting of different grain sizes and composition. The Herbig Ae star HD 34282 shows strong features of PAHs but none of silicate, while the emission spectrum of the Herbig Ae star HD 72106 resembles those of solar-system comets and known Herbig sources of evolved dust. We demonstrate that HD 72106 is host to highly processed silicates and find evidence for enstatite, which is not common in young objects. Evolved dust is also seen in the T Tauri stars HD 98800 and MP Mus. We further detected MP Mus at $1200 \mu \mathrm{m}$ with the bolometer array SIMBA at the SEST in La Silla. The findings of our analysis are given in the context of previous dust studies of young stellar objects.
\end{abstract}

Key words. stars: circumstellar matter - stars: planetary systems: protoplanetary disks - stars: pre-main sequence infrared: stars - techniques: spectroscopic

\section{Introduction}

It is generally believed that circumstellar (CS) disks are required for the formation of stars and planetary systems. Planets are built up by coagulation of dust and gas (e.g. Beckwith et al. 2000). The final stages of these disks remain to be characterised, but observations suggest that protoplanetary accretion disks turn into debris disks at the end of the planet formation phase.

Near-IR studies of CS disks measure the scattered light from dust in the inner disk region, which may strongly depend on the disk structure. At longer wavelengths dust dominates the emission. However, imaging disk searches in the mid-IR are difficult with current ground-based instruments due to limited sensitivity and spatial resolution. The existence of CS matter can more easily, although indirectly, be revealed with photometry and spectroscopy.

In this paper we present a search and analysis of CS matter for a sample of pre-main sequence objects whose IR

* Based on observations collected at the European Southern Observatory, La Silla, Chile (69.C-0073, 70.C-0468, 71.C-0001, 73.C-0372). appearances have not yet been well characterised in the literature. Our goal is to obtain insight into the dust properties by modelling the $N$-band silicate features, and to compare the result with those of other sources.

Cohen \& Witteborn (1985) performed $10 \mu \mathrm{m}$ spectrophotometry of $32 \mathrm{~T}$ Tauri or related young stars and concluded that the CS matter has a disk-like geometry. $N$-band spectroscopy of 23 young stars was shown by Hanner et al. (1998); their sample includes four members of the FU Ori class (FUOR). A new way of modelling the silicate dust features was presented by Bouwman et al. (2001, henceforth B2001) for a sample of 14 Herbig Ae/Be stars (HAeBe). This technique was also used by Meeus et al. (2003) for a group of three T Tauri stars (TTS). In both cases large differences in the amount of dust processing were found in an otherwise fairly homogeneous sample. A correlation between the strength of the silicate feature and its shape, which is interpreted as evidence of grain processing in CS disks, was found by van Boekel et al. (2003). Przygodda et al. (2003) performed a similar analysis for $14 \mathrm{~T}$ Tauri stars and found the same correlation. Meeus et al. (2001, henceforth M2001) analysed 2-45 $\mu \mathrm{m}$ ISO-SWS spectra and spectral energy distributions of $14 \mathrm{HAeBes}$ and explained their differences 
Table 1. Stellar parameters and known fluxes for our target sample. $V$-band magnitudes and the IRAS $12 \mu$ m fluxes (except for V 346 Nor) are taken from the SIMBAD database. $T_{\text {adopted }}$ and $(\log g)_{\text {adopted }}$ list the temperature and gravity which we used to select the appropriate Kurucz atmosphere model for the HAeBe and T Tauri stars (for completeness these values are also shown for the FUOR sources). The mm-fluxes have been measured by (1) Piétu et al. (2003); (2) Stern et al. (1994); (3) This work (at $1200 \mu \mathrm{m}$ ); (4) Reipurth et al. (1993); and (5) Sandell \& Weintraub (2001, given as flux/beam). Stellar ages: (6) From Fig. 4 in Piétu et al. (2003) we roughly estimate the given age. Other values were determined by (7) Prato et al. (2001, PMS evolutionary tracks); (8) Mamajek et al. (2002, spectral characteristics and isochrones); (9) Van den Ancker et al. (2004, comparison with PMS evolutionary tracks). The distances are from (10) Piétu et al. (2003); (11) The Hipparcos Catalogue; (12) Prato et al. (2001); (13) Mamajek et al. (2002); (14) Graham \& Frogel (1985); (15) Sandell \& Weintraub (2001); (16) Ibragimov $\&$ Shevchenko (1990); and (17) Reipurth et al. (2002). The extinction $A_{\mathrm{V}}$ is taken from (18) Acke \& van den Ancker (2004b); (19) Vieira et al. (2003); (20) Sylvester et al. (1996); (21) Mamajek et al. (2002); (22) Gredel (1994); (23) Value taken from Van den Ancker et al. (2004), but adapted to our photometry; (24) Reipurth et al. (2002).

\begin{tabular}{lccrcrrrrrrrrrr}
\hline \hline Object & Class & Spectral type & $\begin{array}{r}T_{\text {adopted }} \\
{[\mathrm{K}]}\end{array}$ & $\begin{array}{l}(\log g)_{\text {adopted }} \\
{[\mathrm{mag}]}\end{array}$ & $\begin{array}{r}V \\
F_{12 \mu \mathrm{m}} \\
{[\mathrm{Jy}]}\end{array}$ & $\begin{array}{r}F_{1300 \mu \mathrm{m}} \\
{[\mathrm{mJy}]}\end{array}$ & $\begin{array}{c}\text { Ref. } \\
{[\mathrm{Mgy}]}\end{array}$ & $\begin{array}{c}\text { Ref. } \\
{[\mathrm{pc}]}\end{array}$ & $\begin{array}{c}\text { Ref. } \\
{[\mathrm{mag}]}\end{array}$ & $\begin{array}{c}A_{\mathrm{V}} \\
\text { Ref. }\end{array}$ \\
\hline HD 34282 & HAeBe & A0 & 10000 & 4.5 & 9.85 & 0.70 & $110 \pm 10$ & $(1)$ & $5-10$ & $(6)$ & 400 & $(10)$ & 0.28 & $(18)$ \\
HD 72106 & HAeBe & A0 IV & 9500 & 4.5 & 8.50 & 2.22 & - & - & - & - & 290 & $(11)$ & 0.0 & $(19)$ \\
HD 98800 & TTS & K5 / K7 / M1 V & 4000 & 4.5 & 9.11 & 1.98 & $36 \pm 7$ & $(2)$ & $7-10$ & $(7)$ & 47.6 & $(12)$ & 0.31 & $(20)$ \\
MP Mus & TTS & K1 IV & 4250 & 4.5 & 10.32 & 0.88 & $224 \pm 8$ & $(3)$ & 8 & $(8)$ & 86 & $(13)$ & 0.17 & $(21)$ \\
V 346 Nor & FUOR & - & 5500 & 4.5 & 16.3 & 7.50 & $273 \pm 15$ & $(4)$ & - & - & 700 & $(14)$ & 2.7 & $(22)$ \\
V 883 Ori & FUOR & - & 5500 & 4.5 & $\sim 15$ & 52.5 & $549 \pm 23$ & $(4)$ & - & - & 460 & $(15)$ & - & - \\
Z CMa & FUOR & - & 30000 & 4.5 & $9-11$ & 126.6 & $446 \pm 16$ & $(4)$ & 0.3 & $(9)$ & 930 & $(16)$ & 3.8 & $(23)$ \\
BBW 76 & FUOR & G0 - G2 I & 5500 & 4.5 & $\sim 12$ & 1.02 & $13.3 \pm 2.4$ & $(5)$ & - & - & 1800 & $(17)$ & 2.1 & $(24)$ \\
\hline
\end{tabular}

in terms of a different disk geometry. Acke \& van den Ancker (2004b) extended this sample, investigating ISO-SWS spectra of 46 HAeBe stars in total. As well as an analysis of the silicate bands around $10 \mu \mathrm{m}$, they focus on the PAH emission and relate its presence to the disk geometry of the objects.

Half of our targets are FU Ori objects. This is a class of low-mass pre-main sequence stars, whose variability is characterised by a dramatic outburst in optical light followed by a fading phase which lasts decades. The origin of this phenomenon is not clear but it is often associated with enhanced accretion (Hartmann \& Kenyon 1996). Herbig et al. (2003), however, proposed a very different interpretation. Recently, the effect of these optical outbursts upon the object's appearance in the infrared has been investigated (Andrews et al. 2004; Ábrahám et al. 2004).

The structure of this paper is as follows: in Sect. 2 we introduce our targets and in Sect. 3 we describe the observations and data reduction. Modelling and analysis of the $N$-band spectra are presented in Sect. 4. We discuss the dust composition of the individual targets in Sect. 5 and finish with a summary and conclusions in Sect. 6. In a second paper a different sample is analysed in a similar way, consisting of Vega-type and post-main sequence targets (Schütz et al. 2005, hereafter called Paper II).

\section{Observed sources}

Our targets are heterogeneous in spectral type and fraction of IR excess, but all objects are still on the pre-main sequence. The sample includes four FU Ori, two T Tauri and two Herbig Ae stars. Their stellar parameters and both IRAS and mm fluxes are given in Table 1 . We note that for many of our targets - besides IRAS photometry - this is their first observation in the mid-IR. For those cases where more mid-IR data have already been published we give references. In the following paragraph the sources are described individually:

- The Herbig Ae star HD 34282 was shown by Sylvester et al. (1996) to have a strong infrared excess towards far-IR wavelengths. Mannings \& Sargent (2000) obtained unresolved mm-measurements with the Owens Valley Radio Observatory. A large, massive Keplerian disk $\left(R_{\text {out }}=835 \pm\right.$ 20 AU) was confirmed by Piétu et al. (2003) using the IRAM Plateau de Bure Interferometer. They also point out that the Hipparcos distance to HD 34282 has been underestimated. $N$-band spectra were published in Sylvester \& Mannings (2000) and Acke \& van den Ancker (2004b), but their quality is not sufficient to allow a detailed dust analysis.

- Not much is known about HD 72106. It is a binary with 0.'78 separation (Fabricius \& Makarov 2000). Optical spectroscopy characterised its southern component as a HAeBe candidate (Vieira et al. 2003).

- HD 98800 (alias TWA 4) represents a visual binary with 0.8 projected separation, whose components each consist of a spectroscopic binary. Gehrz et al. (1999) showed that the large mid-infrared excess is entirely associated with the binary system HD 98800 B, which was later confirmed by Koerner et al. (2000). Prato et al. (2001) explained the excess by a circumbinary disk which is tidally truncated to an outer radius of 10-15 AU, and calculated a blackbody temperature of $150 \mathrm{~K}$ for the dust. In the following sections we always refer to the northern component B when talking about HD 98800. Infrared spectra covering the region around $10 \mu \mathrm{m}$ were already published by Sylvester et al. (1996) and Sitko et al. (2000), but both spectra did not have adequate quality to discuss the mineralogy in detail.

- MP Mus (alias PDS 66) shows both strong $\mathrm{H}_{\alpha}$ emission and $K$-band excess, indicative of accretion. It is the only 
Table 2. Results from TIMMI 2 photometry and spectroscopy are merged in this table. The airmass and TIMMI 2 integration time refer to $N$-band spectroscopy. Mid-IR photometry was obtained either in the N1 $\left(\lambda_{0}=8.6 \mu \mathrm{m}\right)$ or the N11.9 $\left(\lambda_{0}=11.6 \mu \mathrm{m}\right)$ passband. Errors represent the accuracy of this aperture photometry and do not necessarily include the uncertainty which measurements on different nights may introduce due to atmospheric fluctuations (errors caused by the latter one might amount up to $10 \%$ for the fainter sources).

\begin{tabular}{lcccccc}
\hline \hline Object & $\begin{array}{c}\text { Time of } \\
\text { observation }\end{array}$ & Airmass $(N$-spec $)$ & $\begin{array}{c}\mathrm{t}_{\text {int }}(N \text {-spec }) \\
{[\mathrm{min}]}\end{array}$ & $\begin{array}{c}F_{\mathrm{N} 1} \\
{[\mathrm{Jy}]}\end{array}$ & $\begin{array}{c}F_{\mathrm{N} 11.9} \\
{[\mathrm{Jy}]}\end{array}$ & $N$-spec features \\
\hline HD 34282 & Dec. 2002 & $1.2-1.4$ & 67 & - & $0.60 \pm 0.02$ & PAH at 8.7 and 11.2 $\mu \mathrm{m}$ \\
HD 72106 & Mar. 2004 & 1.3 & 25 & - & $2.89 \pm 0.15$ & Silicate emission \\
HD 98800 & Jun. 2002 & 1.1 & 16 & $0.83 \pm 0.09$ & - & Silicate emission \\
MP Mus & Dec. 2002 & $1.5-1.7$ & 49 & - & $0.73 \pm 0.03$ & Silicate emission \\
V 346 Nor & Sep. 2003 & 1.2 & 12 & - & $7.29 \pm 0.07$ & Silicate absorption \\
V 883 Ori & Mar. 2004 & 1.4 & 6 & - & $52.5 \pm 2.6$ & Silicate absorption \\
Z CMa & Mar. 2004 & 1.2 & 6 & - & $183.8 \pm 8.9$ & Silicate absorption \\
BBW 76 & Mar. 2004 & 1.2 & 28 & - & $0.82 \pm 0.05$ & Silicate emission \\
\hline
\end{tabular}

classical TTS among a PMS population of 110 members in an OB association (Mamajek et al. 2002).

- V 346 Nor is considered as a FUOR. Prusti et al. (1993) gave corrected IRAS fluxes for V 346 Nor, since the values shown in the IRAS point source catalogue are contaminated by the close object $\operatorname{Re} 13$.

- V 883 Ori is also classified as FUOR, based upon the similarity of its optical spectrum with that of FU Ori itself (Strom \& Strom 1993). CO near-IR absorption bands support this assumption (Reipurth \& Aspin 1997).

- The FUOR Z CMa is a well-studied source. Koresko et al. (1991) revealed the binarity of Z CMa with a separation of 0.1 . The system probably consists of a B0 IIIe primary and a FUOR-type secondary. Both components have an estimated age of $\sim 3 \times 10^{5} \mathrm{yr}$ (Van den Ancker et al. 2004). Mid-IR spectra were already presented by Cohen \& Witteborn (1985) and Acke \& van den Ancker (2004b). Because of the time-variant nature of FUORs, further spectra would be helpful to investigate the spectral evolution of these objects in their fading and erupting phase.

- A review of the FUOR BBW 76 (alias Bran 76) and its variability at optical and near-IR wavelengths was presented by Reipurth et al. (2002). The object illuminates its surrounding cloud in an extensive reflection nebula.

\section{Observations and data reduction}

The mid-IR observations were carried out during runs in June 2002, December 2002, September 2003 and March 2004 with the ESO TIMMI2 camera $^{1}$ at La Silla observatory. Weather conditions did not permit $Q$-band imaging and we concentrated the photometry mainly on the N11.9 filter (central wavelength $11.6 \mu \mathrm{m}$ ). $N$-band spectra were obtained between 8-13 $\mu \mathrm{m}$ applying a standard chopping and nodding technique along the $3^{\prime \prime}$ slit with a throw of $10^{\prime \prime}$. For spectra, the airmass and on-source integration times are shown in Table 2. Standard stars for telluric correction and flux calibration had been selected from a list of mid-IR standard stars given on the TIMMI 2 webpage ${ }^{2}$ and were observed close in

\footnotetext{
${ }^{1}$ http://www.ls.eso.org/lasilla/sciops/3p6/timmi/

${ }^{2}$ http://www.ls.eso.org/lasilla/sciops/3p6/timmi/ html/stand.html
}

time and airmass $(<0.2$ relative to the targets' airmass). We apply the airmass correction described in Schütz \& Sterzik (2004) and furthermore correlate the spectrophotometric calibration with the obtained photometry. The wavelength calibration is optimised using the atmospheric absorption features of ozone $(9.58 \mu \mathrm{m})$ and $\mathrm{CO}_{2}(11.73 \mu \mathrm{m}, 12.55 \mu \mathrm{m})$ in addition to the given wavelength calibration table. During the observations in 2002 the instrument was set up with a backup detector showing a low and varying response in one detector column. The corresponding area between approximately 9.0 and $9.7 \mu \mathrm{m}$ is removed from the spectra presented in Sect. 4. In the run of September 2003, full spectral coverage was available again, but atmospheric conditions were instable and sometimes left uncorrectable ozone features around $9.5 \mu \mathrm{m}$. By comparing data from different nights and applying various standard stars for comparison, the exact position of the remnant feature is identified and removed from the spectra. Depending on the atmospheric quality, this leaves gaps of different sizes up to a maximum range between 9.4 and $9.7 \mu \mathrm{m}$. In total, the estimated average accuracy of the spectra (with regard to the noise) is better than $3 \%$ for V 346 Nor, V 883 Ori and Z CMa, around 5\% for HD 34282 and HD 72106, 10-15\% for HD 98800 and MP Mus, while for BBW 76 the accuracy is within $20 \%$.

We also observed MP Mus at $1.2 \mathrm{~mm}$ with the bolometer array SIMBA at the SEST in La Silla. Details of these observations are given in Sect. 3 of Paper II. The object was detected with a flux density of $224 \pm 8 \mathrm{mJy}$.

\section{Analysis and modelling results}

Apart from the mid-IR and mm photometry shown in Table 2, we collected fluxes from the literature in the passbands $U B V R I$, $J H K$ (2MASS), 12, 25, 60 and $100 \mu$ m (IRAS) as well as ISO and (sub-)millimeter data - where available. In Figs. 1 and 2 the resulting optical to $\mathrm{mm}$ spectral energy distributions (SED) are shown as an aid for interpreting the TIMMI2 $\mathrm{N}$-band spectra. To emphasise the non-stellar contribution to the SED, we plotted a reddened Kurucz atmosphere model (Kurucz 1994) for the HAeBe and T Tauri stars, using the stellar parameters in Table 1. For the FUOR objects we did not plot a Kurucz model, since in these cases the $V$-band flux most likely arises from the star plus the hot inner part of the outbursting disk. 


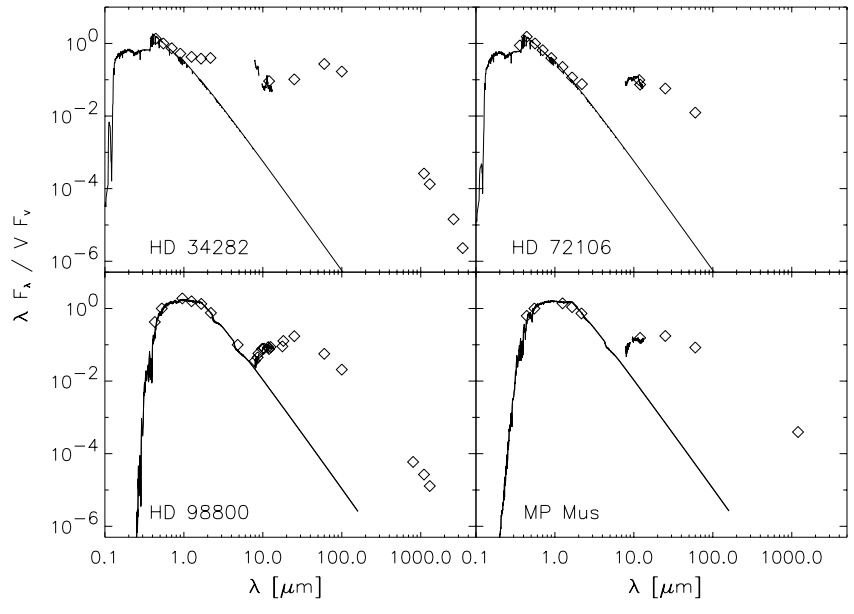

Fig. 1. Optical to millimeter SED for the Herbig Ae and T Tauri stars in our sample, obtained through our observations or collected in the literature. For each source a suitable Kurucz model is overplotted to emphasise the contrast between the photospheric and the CS flux. The position of the TIMMI2 $N$-band spectra is indicated between 8 and $13 \mu \mathrm{m}$ : the agreement with the photometry is excellent. We show the spectra in detail in Figs. 3 and 4.

The disk may be the dominant source and probably has a radial temperature distribution, rather than a single blackbody temperature, thus a single Kurucz model may not be sufficient to describe the contribution of the central source at all wavelengths. Nevertheless, from Figs. 1 and 2 it is recognisable that the excess emission of the FUORs is much larger than those of the HAeBes and TTS, suggesting that the FUORs have a larger amount of CS material.

In the interstellar medium (ISM) the dominant feature at $10 \mu \mathrm{m}$ is caused by small, amorphous silicate grains (Kemper et al. 2004). When stars are formed out of their parental cloud, the material in their disks is assumed to have a similar composition as that found in the ISM. Changes in the size and composition are expected to occur during the subsequent evolution of the object, eventually leading to a planetary system. More precisely, grains start to grow and become crystalline through a process called thermal annealing. Laboratory experiments have shown that, due to thermal annealing, amorphous silicates gradually turn into crystalline forsterite and silica (e.g. Rietmeijer 1989; Hallenbeck \& Nuth 1997). B2001 found a correlation between the amount of forsterite and silica in the disks of HAeBe, showing that thermal annealing indeed takes place in these objects. All the above-mentioned species emit in the $N$ band, making this an excellent window to study dust evolution in the inner parts $(r<10 \mathrm{AU})$ of a protoplanetary disk. The $10 \mu \mathrm{m}$ silicate emission is thought to originate from a surface layer of the inner disk (Chiang \& Goldreich 1997), which needs to be located close to the star as it is too large to be transiently heated, while PAH emission can originate from molecules located further away in the surface layer (also called "atmosphere"), since these are excited by UV photons.

In Figs. 3 and 4 we show $N$-band spectra for our target sample and see that large differences exist between the sources. To quantify these differences and to determine the composition of their CS dust, we adopt the same procedure which was

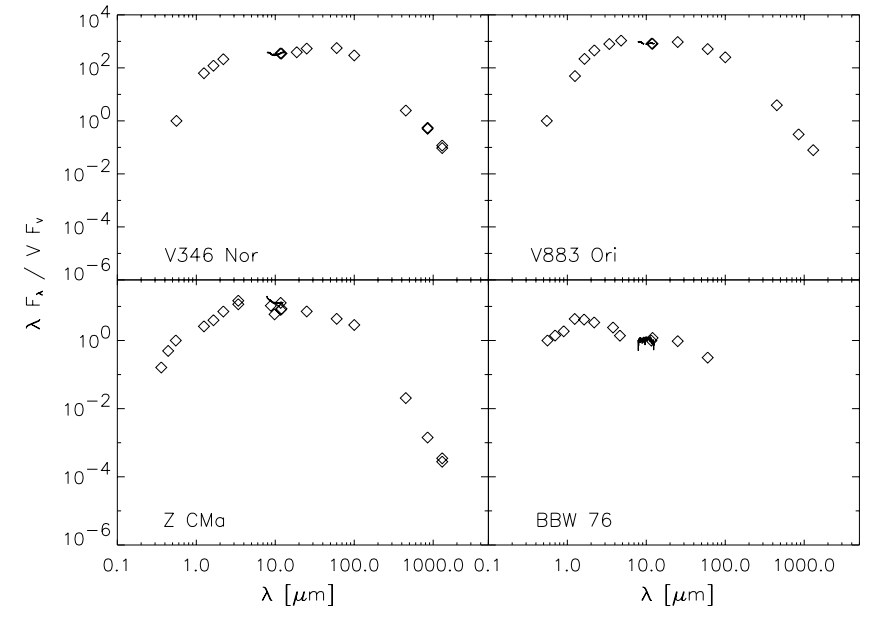

Fig. 2. SEDs for the FUOR objects. No Kurucz model is plotted here as explained in the text.

described by B2001 and Meeus et al. (2003) for interpreting mid-IR spectra of PMS stars. We first determine and subtract a local continuum to our TIMMI 2 spectra by fitting a blackbody to the $8-13 \mu \mathrm{m}$ region. Given the small wavelength range, this fit is not a unique solution but the absolute flux of the features after subtraction is correct within $10 \%$. This uncertainty, however, has only a negligible effect on the derivation of the dust composition. Subsequently, we fit the continuum-subtracted spectra with a linear combination of emission features from the following (dust) species which are commonly found in disks of PMS stars:

- Polycyclic Aromatic Hydrocarbons (PAHs).

- Amorphous olivine $\left([\mathrm{Mg}, \mathrm{Fe}]_{2} \mathrm{SiO}_{4}\right)$ with grain sizes of 0.1 and $2.0 \mu \mathrm{m}$, which we will refer to as "small" and "large" silicate grains. We used absorption coefficients of spherical grains by Dorschner et al. (1995).

- Crystalline silicates: magnesium forsterite $\left(\mathrm{Mg}_{2} \mathrm{SiO}_{4}\right)$ and enstatite $\left(\mathrm{MgSiO}_{3}\right)$. We assumed a continuous distribution of ellipsoids (CDE) for their shape. Coefficients for forsterite are from Servoin \& Piriou (1973), for enstatite from Jaeger et al. (1998).

- Silica $\left(\mathrm{SiO}_{2}\right)$ coefficients, again adopting CDE, were taken from Spitzer \& Kleinman (1961).

We took the absorption coefficients directly from B2001 and refer for a discussion on the influence of the grain shape on the spectroscopic signature to that article. Figure 5 illustrates the wavelength dependence of the above-mentioned emission features. Modelling results for the emission spectra, together with the separate contribution of each dust component are shown in Fig. 6. When deriving in which amounts the different dust species are present, the linear coefficients of the fit - which are proportional to the radiating surface of the grains - need to be converted to mass. However, it is not possible to determine the absolute amount of mass of each species present, as we have no spatially resolved data to derive the particles' size, density or temperature distribution as a function of radius within the disk. Therefore, we derived mass ratios of the different species under the assumption that (1) the particles are spherical; and (2) the 

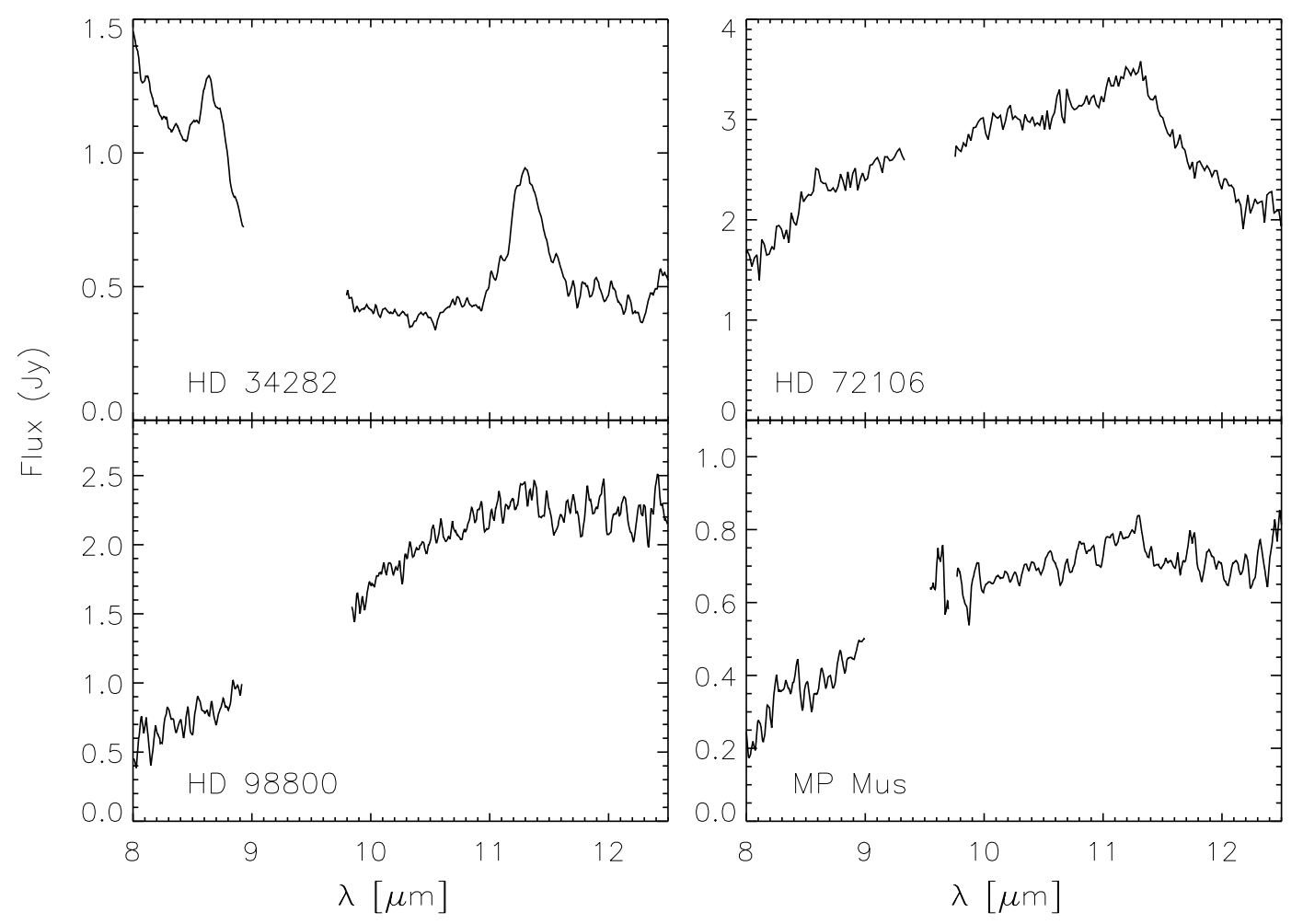

Fig. 3. TIMMI2 spectra of the Herbig Ae and T Tauri stars. See the text for a discussion of the individual targets. For some objects, the data between approximately 9.0 and $9.7 \mu \mathrm{m}$ has been removed, as there was a detector problem in 2002. Smaller gaps appear between approximately 9.4 and $9.7 \mu \mathrm{m}$, where we cut out uncorrectable atmospheric ozone features (cf. text).
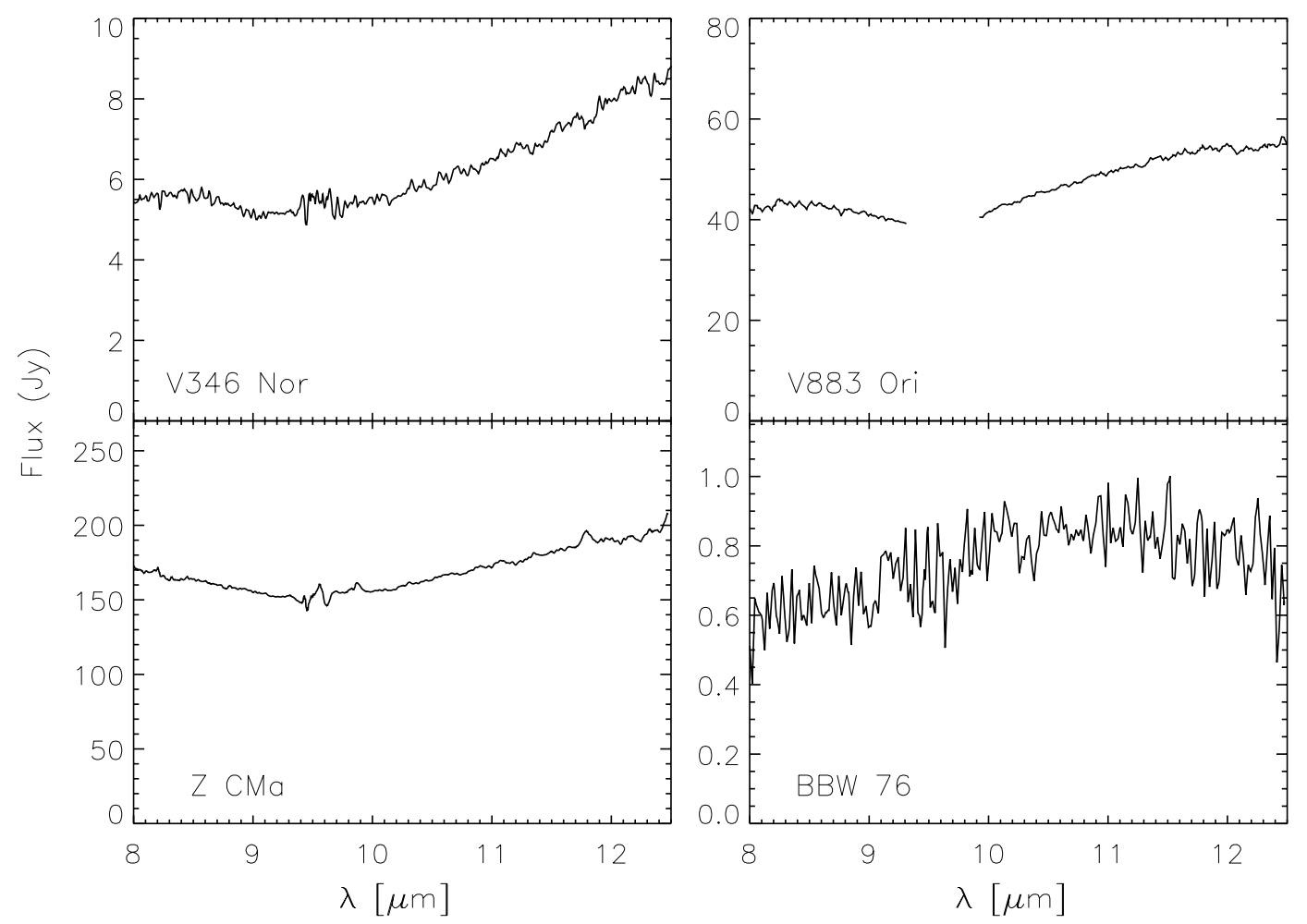

Fig. 4. TIMMI2 spectra for the FUOR targets. See the caption of Fig. 3 for further details. The objects V 346 Nor, V 883 Ori and Z CMa show an absorption feature, pointing to optically thick material, while BBW 76 shows an emission feature, pointing to optically thin material in the line of sight. 


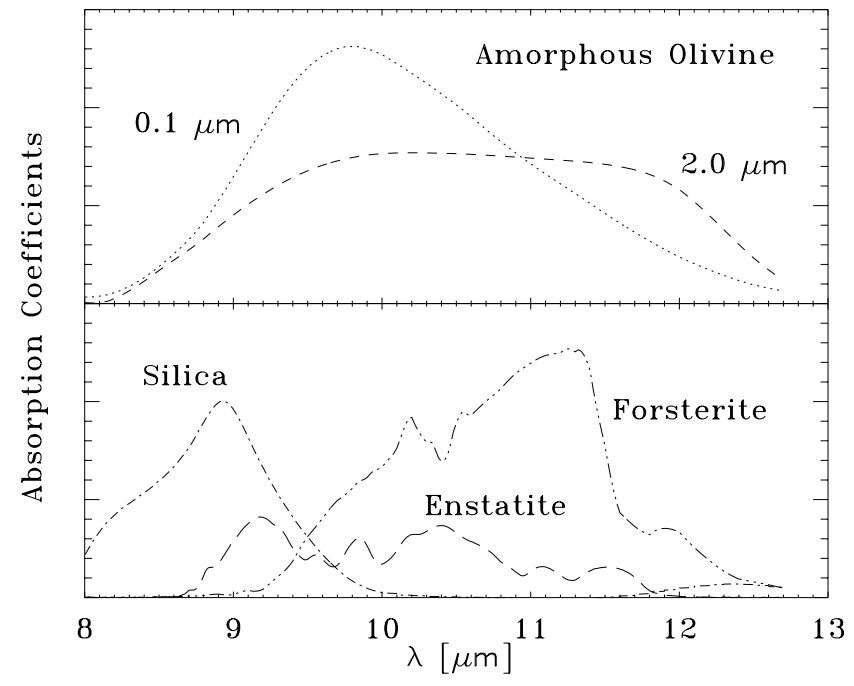

Fig. 5. Emission features of the modelled silicate dust grains. The relative absorption coefficients are plotted against wavelength. Top panel: absorption coefficients for large (dashed curve) and small (dotted) amorphous olivine. Bottom panel: silica $\left(\mathrm{SiO}_{2}-\right.$ dash-dotted $)$, crystalline forsterite (dash plus three dots) and enstatite (long dashes).

Table 3. Mass ratios derived from the model fits of the $N$-band spectra: large to small amorphous silicates $\left(m_{2.0} / m_{0.1}\right)$, forsterite to amorphous silicates $\left(m_{\mathrm{forst}} / m_{\mathrm{sil}}\right), \mathrm{SiO}_{2}$ to silicates $\left(m_{\mathrm{SiO}_{2}} / m_{\mathrm{sil}}\right)$ and enstatite to silicates $\left(m_{\text {enst }} / m_{\text {sil }}\right)$.

\begin{tabular}{lcccc}
\hline \hline Object & $m_{2.0} / m_{0.1}$ & $m_{\text {forst }} / m_{\text {sil }}$ & $m_{\mathrm{SiO}_{2}} / m_{\text {sil }}$ & $m_{\text {enst }} / m_{\text {sil }}$ \\
\hline HD 72106 & Only large & 1.03 & 0.33 & 0.48 \\
HD 98800 & 12.0 & 0.38 & 0.04 & - \\
MP Mus & 5.3 & 0.26 & 0.11 & - \\
\hline
\end{tabular}

particles have the same density. The mass ratios are meaningful to compare the objects in our sample and to establish the amount of processed dust we observe at $10 \mu \mathrm{m}$. In Table 3 we list the derived mass ratios. Apart from $m_{2.0} / m_{0.1}$, which gives the mass ratio between large and small amorphous grains, the mass ratios always compare the mass of a particular species (forsterite, $\mathrm{SiO}_{2}$ or enstatite) with the total mass in amorphous silicates (both small and large). Please note that for a given mass, small particles have a larger total emitting surface than large ones. Inversively, a similar amount of observed radiation will result in a much smaller mass when caused by small grains than if it was caused by larger grains.

\section{Discussion}

Below we discuss for each object the result of our spectral decomposition and what could be derived from the appearance of its SED. It is important to repeat that our spectral results are only valid for the warm dust, which is located in the inner parts of the disk $(r<10 \mathrm{AU})$.

\subsection{The HAeBe and T Tauri stars}

- HD 34282 shows very strong PAH features located at 8.7 and $11.2 \mu \mathrm{m}$. The peak at $8.7 \mu \mathrm{m}$ is part of the $7-9 \mu \mathrm{m}$ complex. There is an indication that the spectrum rises shortward of the displayed wavelength range towards the $7.7 \mu \mathrm{m}$ PAH feature. Contrary to what is observed in other young sources (Hony et al. 2001), the $11.2 \mu \mathrm{m}$ line is fairly symmetric.

We do not observe a silicate feature for this young HAe star, which can be explained if the silicate grains located in the inner parts of the disk are either (1) too cold; or (2) too large to emit at $10 \mu \mathrm{m}$, as was shown for the HAe star HD 100453 (Meeus et al. 2002). Acke et al. (2004a) calculated a relatively shallow mm spectral index $n$ of 3.36 (where $\lambda F_{\lambda} \propto \lambda^{-n}$, and $n=3$ for a blackbody), which indicates the presence of larger, cold grains in the outer disk. This result is confirmed by Natta et al. (2004), who derive maximum grain sizes up to a few $\mathrm{mm}$, based on $\mathrm{mm}$ observations. However, this reveals no information about the size distribution of the warm dust grains. Nevertheless, we suggest that also those grains have grown substantially: as shown above, the alternative to large grains would be grains with a too low temperature to radiate at $10 \mu \mathrm{m}$. A lack of warm silicate grains could only occur if these grains were shielded from the stellar radiation, e.g. by an inner puffed-up region as suggested by Dullemond et al. (2001). However, as is further discussed towards the end of this subsection, prominent PAH emission is not observed in such self-shielded disks. Thus, we conclude that the small silicate grains in the inner disk region must be strongly depleted, which was also the outcome of passive disk modelling by Dominik et al. (2003).

- The spectrum of HD $\mathbf{7 2 1 0 6}$ is devoid of small, amorphous silicate grains and is dominated by the crystalline silicates forsterite and enstatite. Also large amorphous silicates and $\mathrm{SiO}_{2}$ are present. All these species are the result of dust processing within the CS disk. Until now, such a large fraction of processed dust has only been observed in the Herbig Be stars HD 100546 and HD 179218, making this object an important one for future studies in the context of planet searches.

Malfait et al. (1998) were the first to compare the spectra of the HAeBe star HD 100546 with that of comet Hale-Bopp, finding a strong similarity. They suggested that both objects would have similar dust characteristics. In the upper panel of Fig. 7 we compare HD 72106 with a linear combination of the spectra for two solar-system comets: Hale-Bopp (Crovisier et al. 1997) and Halley (Bregman et al. 1987). The resemblance is remarkable, while the remaining deviation at 8.7 and $11.2 \mu \mathrm{m}$ might be due to the presence of PAHs in HD 72106. This similarity suggests that its inner disk is filled by comet debris, similar to those found in our own solar system. In the lower panel of Fig. 7 we compare HD 72106 with the Herbig Be object HD 179218, the only star for which the presence of enstatite is robustly derived (B2001). Interestingly, a high mass ratio of enstatite in comet Halley was also derived by the same authors. Enstatite can form when forsterite and silica are being annealed (Rietmeijer 1989). It is not clear why this process does not seem to occur in other objects. B2001 suggest that the presence of enstatite might be due to the high 

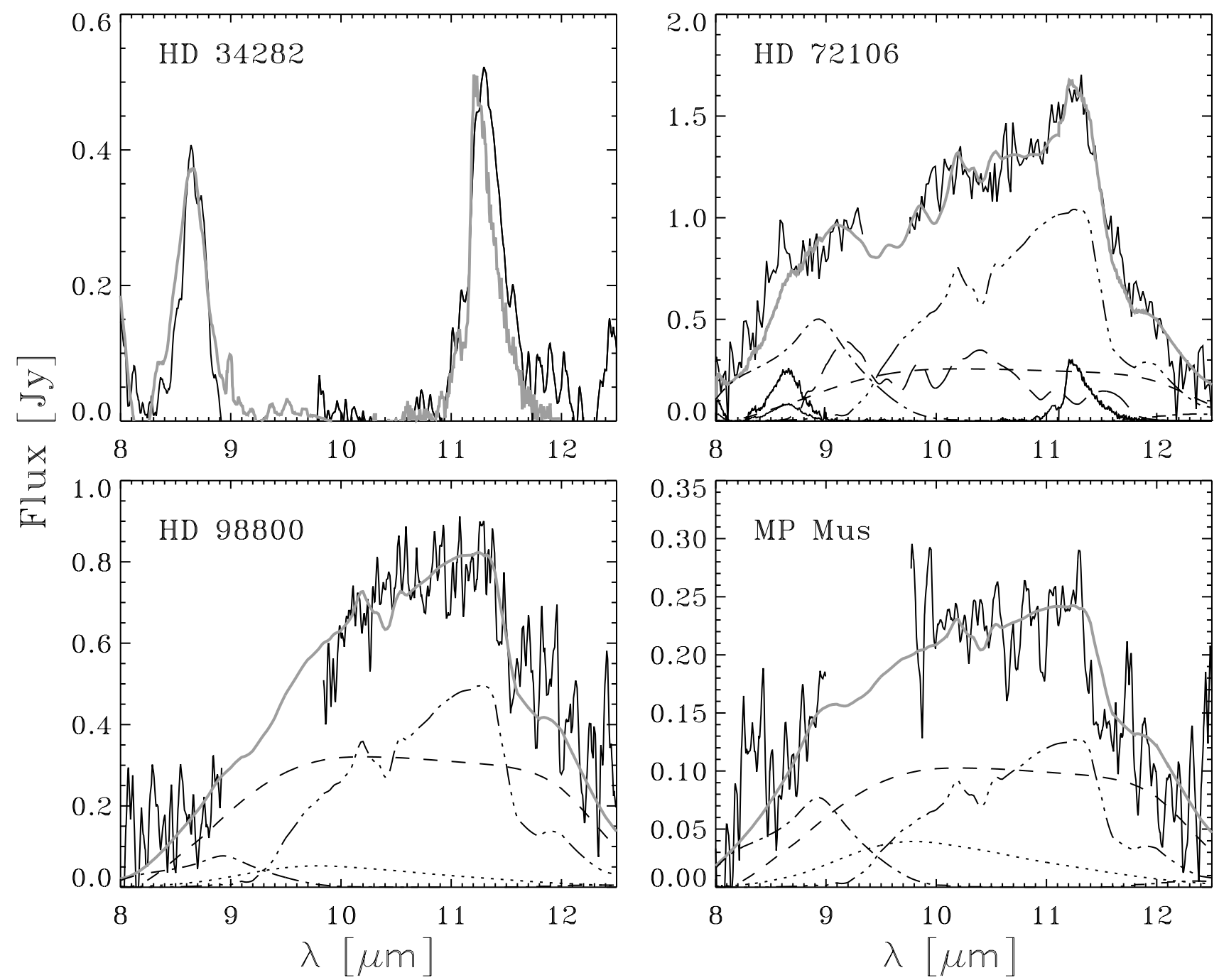

Fig. 6. Continuum subtracted spectra of the PMS stars. Upper left panel: comparison of HD 34282 (thin black curve) with the PAH features of BD+30 3639 (thick grey curve), a well-known source of PAHs (Hony et al. 2001). The PAH features at 8.7 and $11.2 \mu \mathrm{m}$ are clearly visible and agree in position. Other panels: decomposition of the $10 \mu \mathrm{m}$ feature as described in the text. Same linestyles are used for the silicate components as in Fig. 5.

luminosity of HD 179218 which gives rise to rapid dispersal of the gas. Interestingly, the SIMBAD database states a luminosity class IV for HD 72106.

- The $10 \mu \mathrm{m}$ spectrum of HD $\mathbf{9 8 8 0 0}$ was already shown by Sylvester et al. (1996) and later by Sitko et al. (2000), but both spectra did not have adequate quality to discuss the mineralogy in detail. Sylvester et al. (1996), however, noticed that the spectrum peaks longwards of $10 \mu \mathrm{m}$, which is typical for larger silicate grains. Our decomposition of the silicate feature indicates highly processed dust, dominated by both large amorphous olivine and crystalline forsterite. Small silicates have almost disappeared and we found no indication for the presence of enstatite.

- MP Mus: Grains have grown also in this disk and both large amorphous olivine as well as crystalline forsterite are found, while small olivine grains and $\mathrm{SiO}_{2}$ are present in smaller amounts. Our mm observation suggests grain growth, but we need more mm data points to confirm this.
The Herbig Ae stars HD 34282 and HD 72106 both are spectral type A0 and should have a similar level of UV flux. However, the strength of their PAH features is much stronger for HD 34282. Following M2001 and Acke \& van den Ancker (2004b), we can relate this difference to their disk geometry. M2001 classified the HAeBes into two groups, based upon the shape of their SED. Group I objects have a rising mid-IR SED, which can be fitted with a power-law and a blackbody continuum and is attributed to a flared disk geometry. Group II objects show a smaller mid-IR excess, their SED can be explained with a power-law and is related to a flat disk geometry. This geometrical model was later confirmed by Dullemond (2002), who made a self-consistent model and showed that Group I sources have flared disks, while representatives of Group II possess flat self-shadowed disks. M2001 noted that the strongest PAH emission occurs in Group I sources, while it is much weaker or even absent in Group II. This was confirmed by Acke \& van den Ancker (2004b) for a larger sample of 


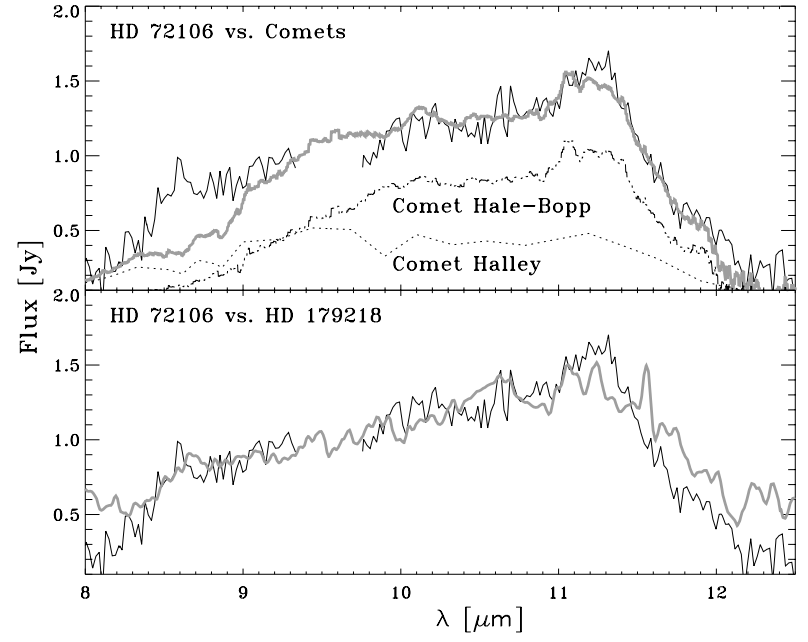

Fig. 7. Top panel: comparison of the Herbig Ae star HD 72106 (solid black curve) with a combination of the comets Halley and Hale-Bopp (thick grey line, scaled intensity). The resemblance is remarkable and has, among PMS objects, only been found for the Herbig Be star HD 100546. Bottom panel: comparison with the Herbig Be star HD 179218. This object was - until now - the only known stellar host of enstatites.

46 HAeBes. In the flared disks, PAHs residing in the disk atmosphere are easily hit by UV photons, while in a self-shadowed disk the thick inner disk rim might intercept those UV photons, giving rise to weaker or even absent PAH emission. Indeed, HD 34282 is a member of Group I, while HD 72106 is a member of Group II, what can explain the differences in PAH strength.

\subsection{The FU Ori sources}

FU Ori objects are most likely still in a - non-steady - accreting phase and embedded in (part of their) parental cloud. Therefore, we expect to see absorption features rather than emission in the infrared, although Hanner et al. (1998) already found FUORs with silicate emission. Given the large variability of FUORs in the optical, attributed to accretion events, studies of these variations in the infrared have meanwhile begun (Ábrahám et al. 2004).

- The SEDs of V 346 Nor, V 883 Ori and Z CMa show a large infrared to millimeter excess, pointing to the presence of a circumstellar envelope enshrouding these objects. Superimposed on the dust continuum emission, the $10 \mu \mathrm{m}$ silicate feature appears in absorption. In Fig. 8 we compare the absorption features of these targets with a source of amorphous silicates, the interstellar medium. For this purpose, we use the observed absorption of the ISM in the direction of the galactic centre ( $\operatorname{Sgr} \mathrm{A}^{*}$ ) and follow the method by Kemper et al. (2004) to convert the absorption feature into optical depth. The spectrum of V 346 Nor agrees well with that of the ISM, while for the other two FUORs we see larger differences. V 883 Ori seems to have extra emission on top of its absorption, while $\mathrm{Z} \mathrm{CMa}$ appears to have larger silicates than seen in the ISM. It is

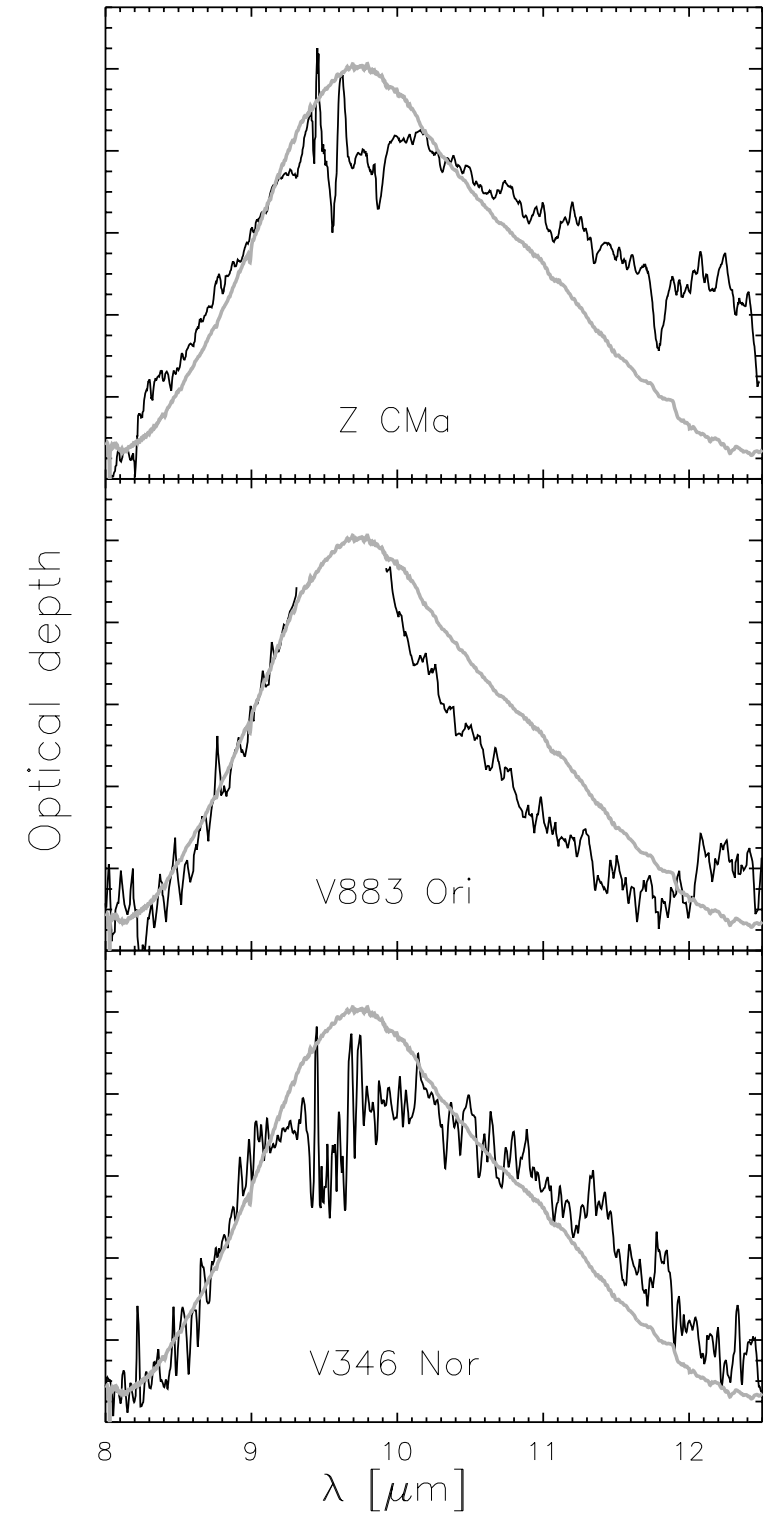

Fig. 8. The optical depth towards V 346 Nor, V 883 Ori and Z CMa is compared with the optical depth towards the galactic center, towards the galactic center (grey curve). For further details, see the text. The features around $9.5 \mu \mathrm{m}$ were caused by variable ozone emission.

interesting to note in this context that Acke \& van den Ancker (2004b) claim to see crystalline silicates in absorption for $\mathrm{Z} \mathrm{CMa}$, which does not disagree with our spectrum, as we see a tentative crystalline feature around $11.3 \mu \mathrm{m}$.

In Fig. 9 we compare the spectral shapes of V 346 Nor and Z CMa obtained with TIMMI2 to ISO-SWS data from August 1996 (V 346 Nor) and November 1997 (Z CMa). The ISO spectrum of V 346 Nor was obtained from the ISO data archive and has not been published before. We had to shift the TIMMI2 spectrum of Z CMa downwards by $75 \mathrm{Jy}$ to match the ISO-SWS spectrum, most likely due to the variability of this target. Within the errors, the spectral shapes agree. We further compare this steady behaviour in the $\mathrm{N}$-band with optical photometry of Z CMa by van den Ancker et al. (2004). As therein no data for 2004 


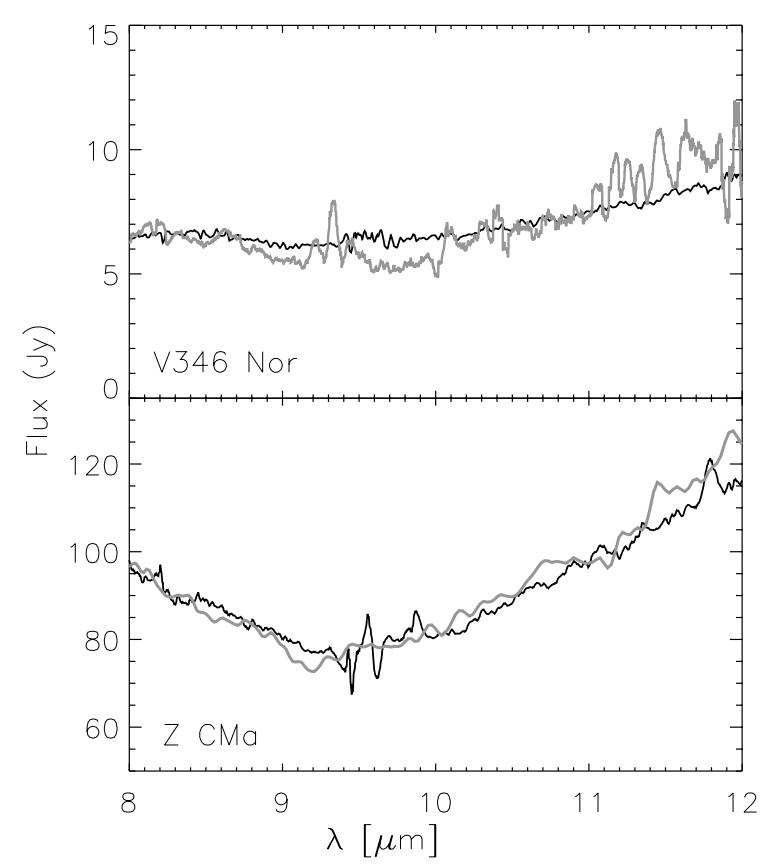

Fig. 9. Comparison of the spectral shapes for V 346 Nor and Z CMa at different epochs. Dark line: data from this work. Grey line: ISO-SWS data are taken on August 31, 1996 (V 346 Nor) and November 7, 1997 ( $\mathrm{Z} \mathrm{CMa).} \mathrm{The} \mathrm{spectral} \mathrm{shapes} \mathrm{although} \mathrm{taken} \mathrm{at} \mathrm{different} \mathrm{epochs} \mathrm{agree}$ with each other.

is included yet, we refer to the year 2003. After a period of slow fading, the object became brighter in 1999 during a six month period, after which it faded again to its previous brightness. Between the two spectral observations, it increased by a few tenths of a magnitude, but no large eruption has been observed. For V 346 Nor we did not find optical data in the required timespan, while during the period 1980-1998 the object became brighter in the $K$-band (Ábrahám et al. 2004). We thus do not dispose of enough material yet to draw any conclusions concerning the optical variability in correlation with the IR spectral variability.

- BBW 76: Contrary to the three other FUORs, this object shows a smaller fraction of IR excess, suggesting that it is less embedded than the other three. Also the absence of an absorption feature, caused by optically thick material, confirms this suspicion. Is this object in a different (accretion) stage than the other FUORs? It may be an interesting target for further studies, but not unique, as Hanner et al. (1998) already found FUORs with silicate emission. We did not model the spectrum, as it has a low quality, but its shape suggests that PAHs and crystalline features are not present.

A follow-up study, observing these objects at several different epochs, together with simultaneous optical photometry is necessary to establish the cause of the FUOR phenomenon.

\subsection{Comparison with previous studies}

The method we used to interpret our $10 \mu \mathrm{m}$ spectra was - to our knowledge - only used by B2001 (who developed the method), Meeus et al. (2003), Przygodda et al. (2003) and Honda et al. (2003). We will refer mainly to those studies to compare our results.
B2001 analysed the $10 \mu \mathrm{m}$ spectra of 14 optically visible HAeBes and found large differences between the objects: some were dominated by amorphous silicate grains (e.g. AB Aur), others were rich sources of crystalline silicates (e.g. HD 100546). Of the two HAeBes in our sample, only HD 72106 shows a silicate emission feature. This source is dominated by large and crystalline grains and is similar to HD 179218 and HD 100546, which also stood out in the study of B2001. HD 72106 is thus a source of highly processed dust, which is not often observed in the group of known HAeBes, and can be considered as a very interesting object in the framework of dust evolution. It is the second known source of enstatite (after HD 179218). The presence of this dust species might be related to a high luminosity of the central sources. M2001 found in their sample of 14 HAeBes four objects which did not show silicate emission, Acke \& van den Ancker (2004b) report a non-detection of this feature for 16 of their 46 sources. We can conclude that the absence of the feature in HD 34282 is not uncommon for a Herbig star.

Meeus et al. (2003) analysed three T Tauri stars in Cha I (age $\sim 1 \mathrm{Myr}$ ) and found a similar variety among those objects: CR Cha is dominated by amorphous silicates, while VW Cha is a source of large, crystalline silicates. The anti-correlation between silicate grain size and strength of the emission band, as found by van Boekel et al. (2003) for a sample of HAeBes, was confirmed by Meeus et al. (2003), a result that was repeated for a larger sample of 16 TTS by Przygodda et al. (2003). Meeus et al. (2003) also found a correlation between the amounts of silica and forsterite, as observed by B2001 for their sample of HAeBes. It was concluded that the dust properties of TTS are very similar to those observed in HAeBes. Honda et al. (2003) studied the 1-10 Myr old TTS Hen3-600 and showed that its dust emission consists mainly of large and crystalline silicates. Our two TTS are also dominated by large and crystalline silicates and possess more evolved dust than the Cha I sample. It is tempting, based upon the findings of this paper and the above-mentioned TTS studies, to attribute the presence of highly evolved dust to a higher age (7-10 Myr), but the sample is still too small to derive a firm conclusion. A larger number of TTS with known ages need to be studied for this purpose. Also, a relation between the amount of dust processing and age has not been established for $\mathrm{HAeBe}$ stars.

\section{Summary and conclusions}

We presented spectral energy distributions and $N$-band spectra of eight PMS stars surrounded by a circumstellar disk, and analysed the dust composition of the spectral emission sources by fitting the observed spectra with known spectral features of different dust species and PAHs. Our sample is too small and heterogeneous to look for a correlation between the dust properties and the objects' properties, therefore we concentrated on each source individually.

The T Tauri and Herbig stars show a SED and emission features in qualitative agreement with the model of a flared or self-shadowing disk surrounded by an optically thin, hot surface layer from which the emission features originate (Chiang \& Goldreich 1997; Dullemond 2002). Strong PAH features are 
found for HD 34282 but no silicate emission, which indicates that small silicates are strongly depleted in this disk. No clear features of PAH are detected in HD 72106, what we attribute to the geometry of the disk which is probably self-shadowed. The most important result of this work is the dust composition of HD 72106, a host of highly processed silicates, similar to those seen in solar-system comets. We also detected enstatite in HD 72106, which is a rare finding in CS disks and might point to rapid gas dispersal due to a higher luminosity of the central star. This target may be a good candidate for extra-solar planet searches, given its similarity to spectra of solar-system comets. Processed dust is also found around the relatively old T Tauri stars (7-10 Myr) HD 98800 and MP Mus, but no enstatite was seen in these objects.

For the FU Ori targets, we observe absorption features in three of the four cases, which is expected for objects embedded in optically thick material. One of the targets - BBW $76-$ shows the silicate feature in emission and is likely less embedded. Multi-epoch simultaneous observations from the optical to the mid-IR are required to study their disk structure and the mechanisms driving their variability.

Acknowledgements. We want to thank B. Acke for useful discussions and for providing the ISO-SWS spectrum of $\mathrm{Z} \mathrm{CMa,} \mathrm{and} \mathrm{also}$ thank the anonymous referee for his/her comments to improve the paper. G.M. acknowledges financial support by the EC-RTN on "The Formation and Evolution of Young Stellar Clusters". This work made usage of the SIMBAD astronomical database and ADS, Strasbourg. We thank the La Silla staff and telescope operators for support during the observations.

\section{References}

Ábrahám, P., Kóspál, Á., Csizmadia, Sz., et al. 2004, A\&A, 428, 89 Acke, B., van den Ancker, M. E., Dullemond, C. P., van Boekel, R., \& Waters, L. B. F. M. 2004a, A\&A, 422, 621

Acke, B., \& van den Ancker, M. E. 2004b, A\&A, 426, 151

Andrews, S. M., Rothberg, B., \& Simon, T. 2004, ApJ, 610, L45

Beckwith, S. V. W., Henning, Th., \& Nakagawa, Y. 2000, in Protostars and Planets IV, ed. V. Mannings, A. P. Boss, \& S. S. Russell (Tucson: University of Arizona Press), 533

Bouwman, J., Meeus, G., de Koter, A., et al. 2001, A\&A, 375, 950 [=B2001]

Bregman, J. D., Witteborn, F. C., Allamandola, L. J., et al. 1987, A\&A, 187,616

Chiang, E. I., \& Goldreich, P. 1997, ApJ, 490, 368

Cohen, M., \& Witteborn, F. C. 1985, ApJ, 294, 345

Crovisier, J., Leech, K., Bockelée-Morvan, D., et al. 1997, Science, 275, 1904

Dominik, C., Dullemond, C. P., Waters, L. B. F. M., \& Walch, S. 2003, A\&A, 398, 607

Dullemond, C. P., Dominik, C., \& Natta, A. 2001, ApJ, 560, 957

Dullemond, C. P. 2002, A\&A, 395, 853

Dorschner, J., Begemann, B., Henning, Th., Jaeger, C., \& Mutschke, H. 1995, A\&A, 300, 503

Fabricius, C., \& Makarov, V. V. 2000, A\&A, 356, 141

Gehrz, R. D., Smith, N., Low, F. J., et al. 1999, ApJ, 512, L55

Graham, J. A., \& Frogel, J. A. 1985, ApJ, 289, 331

Gredel, R. 1994, A\&A, 292, 580

Hallenbeck, S., \& Nuth, J. 1997, Ap\&SS, 255, 427

Hanner, M. S., Brooke, T. Y., \& Tokunaga, A. T. 1998, ApJ, 502, 871
Hartmann, L., \& Kenyon, S. J. 1996, ARA\&A, 34, 207

Herbig, G. H., Petrov, P. P., \& Duemmler, R. 2003, ApJ, 595, 384

Honda, M., Kataza, H., Okamoto, Y. K., et al. 2003, ApJ, 585, L59

Hony, S., Van Kerckhoven, C., Peeters, E., et al. 2001, A\&A, 370, 1030

Ibragimov, M. A., \& Shevchenko, V. S. 1990, in Conf. on Classical Be stars and Ae/Be Herbig Stars, ed. A. Kh. Mamatkazina (Alma Ata)

Jaeger, C., Molster, F. J., Dorschner, J., et al. 1998, A\&A, 339, 904

Kemper, F., Vriend, W. J., \& Tielens, A. G. G. M. 2004, ApJ, 609, 826

Koerner, D. W., Jensen, E. L. N., Cruz, K. L., Guild, T. B., \& Gultekin, K. 2000, ApJ, 533, L37

Koresko, C. D., Beckwith, S. V. W., Ghez, A. M., Matthews, K., \& Neugebauer, G. 1991, AJ, 102, 2073

Kurucz, R. L. 1994, Solar abundance model atmospheres for 0, 1, 2 , 4, $8 \mathrm{~km} \mathrm{~s}^{-1}$, CD-ROM No. 19 (Cambridge, Mass.: Smithsonian Astrophysical Observatory)

Malfait, K., Waelkens, C., Waters, L. B. F. M., et al. 1998, A\&A, 332, L25

Mamajek, E. E., Meyer, M. R., \& Liebert, J. 2002, AJ, 124, 1670

Mannings, V., \& Sargent, A.I. 2000, ApJ, 529, 391

Meeus, G., Waters, L. B. F. M., Bouwman, J., et al. 2001, A\&A, 365, $476[=\mathrm{M} 2001]$

Meeus, G., Bouwman, J., Dominik, C., Waters, L. B. F. M., \& de Koter, A. 2002, A\&A, 392, 1039

Meeus, G., Sterzik, M., Bouwman, J., \& Natta, A. 2003, A\&A, 409, L25

Natta, A., Testi, L., Neri, R., Shepherd, D. S., \& Wilner, D. J. 2004, A\&A, 416, 179

Piétu, V., Dutrey, A., \& Kahane, C. 2003, A\&A, 398, 565

Prato, L., Ghez, A. M., Piña, R. K., et al. 2001, ApJ, 549, 590

Prusti, T., Bontekoe, Tj. R., Chiar, J. E., Kester, D. J. M., \& Whittet, D. C. B. 1993, A\&A, 279, 163

Przygodda, F., van Boekel, R., Àbrahàm, P., et al. 2003, A\&A, 412, L43

Reipurth, B., Chini, R., Krugel, E., Kreysa, E., \& Sievers, A. 1993, A\&A, 273, 221

Reipurth, B., \& Aspin, C. 1997, AJ, 114, 2700

Reipurth, B., Hartmann, L., Kenyon, S. J., Smette, A., \& Bouchet, P. 2002, AJ, 124, 2194

Rietmeijer, F. J. M. 1989, in Lunar and Planetary Science Conference, 19th, Proceedings, A89-36486, 15-91 (Cambridge University Press / Lunar and Planetary Institute), 513

Sandell, G., \& Weintraub, D. A. 2001, ApJS, 134, 115

Schütz, O., \& Sterzik, M. 2004, in High Resolution Infrared Spectroscopy in Astronomy, ed. H. U. Käufl, R. Siebenmorgen, \& A. Moorwood (Springer), in press [arXiv: astro-ph/0404200]

Schütz, O., Meeus, G., \& Sterzik, M. 2005, A\&A, 431, 175

Servoin, J. L., \& Piriou, B. 1973, Phys. Stat. Sol. B, 55, 677

Sitko, M. L., Lynch, D. K., \& Russel, R. W. 2000, AJ, 120, 2609

Spitzer, W. G., \& Kleinman, D. A. 1961, Phys. Rev., 121, 1324

Stern, S. A., Weintraub, D. A., \& Festou, M. C. 1994, IAU Circ., 6003, 3

Strom, K. M., \& Strom, S. E. 1993, ApJ, 412, L63

Sylvester, R. J., Skinner, C. J., Barlow, M. J., \& Mannings, V. 1996, MNRAS, 279, 915

Sylvester, R. J., \& Mannings, V. 2000, MNRAS, 313, 73

van Boekel, R., Waters, L. B. F. M., Dominik, C., et al. 2003, A\&A, 400, L21

Van den Ancker, M. E., Blondel, P. F. C., Tjin A Djie, H. R. E., et al. 2004, MNRAS, 349, 1516

Vieira, S. L. A., Corradi, W. J. B., Alencar, S. H. P., et al. 2003, AJ, 126, 2971 